\title{
Las limitaciones de la Ley de Acceso a la Información en Brasil: reflexiones teóricas en el ámbito de la ciencia de la información
}

\section{Ellen Valotta Elias Borges ${ }^{I}$}

https://orcid.org/0000-0002-7811-0256

\section{Daniel Martínez-Ávila ${ }^{I I}$}

https://orcid.org/0000-0003-2236-553X

\section{Mariana Rodrigues Gomes de Mello ${ }^{I I I}$}

https://orcid.org/0000-0002-5925-8554

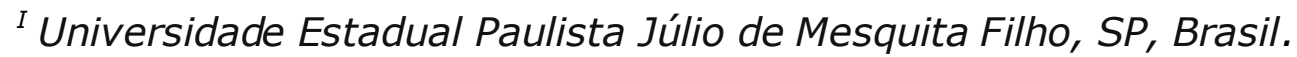
Doutora em Ciência da Informação.

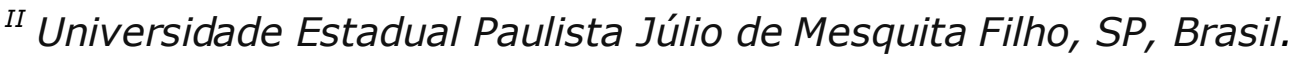
Docente na Universidade Estadual Paulista Júlio de Mesquita Filho.

III Universidade Estadual Paulista Júlio de Mesquita Filho, SP, Brasil. Mestranda no Programa em Ciência da Informação.

\section{http://dx.doi.org/10.1590/1981-5344/3906}

El objetivo de este artículo es verificar la aplicabilidad de la Ley de Acceso a la Información (LAI) en Brasil. Se realizó un análisis comparativo entre la propuesta de Ley y la actuación de los órganos públicos. Se utilizó el servicio electrónico de atención al ciudadano (e-SIC) y el portal del Gobierno Federal. Las solicitudes sometidas a las autoridades competentes permitieron el análisis de la aplicabilidad de la ley. Los resultados mostraron grandes divergencias en cuanto al contenido de la ley. Además, se señala que los estudios sobre la información en el ámbito científico pueden contribuir al desarrollo social cuando se considera no sólo el acceso sino también el proceso de 
apropiación de la información. Se considera que las discusiones sobre el acceso y la apropiación de la información de un modo conjunto pueden traer grandes beneficios tanto para el ámbito científico del área como para el desarrollo social de la población.

Palabras clave: Ley de Acceso a Información; Apropiación de la información; Autoridades competentes de la LAI; Servicio electrónico de atención al ciudadano.

\section{Limits of Freedom of Information Act in Brazil: theoretical reflections in the sphere of information science}

This paper aims to verify the applicability of the Freedom of Information Act (LAI) in Brazil. We conducted a comparative analysis of the proposal of the Law and its applicability by public organisms. We worked with the electronic Citizens' Enquiry Service (e-SIC) and the Federal Government website.. The information requests submitted to the competent law enforcement authorities allowed the analysis of the applicability of the Law. The results showed that there are wide great divergences regarding the content of the law. In addition, they also suggest that the discussions on information, when considering not only information access but also the appropriation of information, can contribute to social development. The consideration of information access and information appropriation in a complementary way may bring significant contributions to the scientific and social fields.

Keywords: Freedom of Information Act; Appropriation of information; Competent law enforcement Authorities; Electroniccitizens' enquiryservice.

Recebido em 14.03.2019 Aceito em 03.04.2020 


\section{Introducción}

La Ley de Acceso a la Información (Lei no 12.527/2011 - LAI), que regula el derecho de acceso a la información pública en Brasil,fue creadael 18 de noviembre de 2011. Esta ley entró en vigor el 16 de mayo de 2012 y establece que toda persona tiene derecho de recibir informaciones de todo órgano público. La ley es válida para los tres poderes de la Unión y fue reglamentada por el Decreto 7.724/2012. De hecho, la aprobación de la Ley ha supuesto un notable cambio en relación a la actuación dediversos organismos públicos, los cuales han demostrado una significanterestructuración y han realizado esfuerzos paramejorar su capacidad interactiva de ofrecer información al ciudadano utilizando tecnologías de alto nivel. De esta forma,la presente investigación se realizó utilizando el sistema electrónico de la administración que posibilita el acceso y contacto con todos los órganos públicos brasileños registrados en dicho sistema electrónico. El uso de este sistema posibilitó la obtención de casi todas las respuestas necesarias $y$, a través del log de ejecución para cada solicitud de información creado por el sistema, el seguimiento y retorno de las solicitudes realizadas en el plazo fijado por la ley.

El principio básico de la LAI es el acceso: "El acceso es la regla, el sigilo es la excepción". En este sentido, debe destacarseel hecho de que aunque todos los ciudadanos tengan derecho al acceso eso no necesariamente implica que todos consiguen comprender la información de la que disponen, es decir, hace falta una apropiación de la información satisfactoria. Batista (2014) señala la dificultad de la aplicabilidad de las leyes y destaca la importancia de la apropiación para el ejercicio democrático pleno, ya que el derecho a la información no sólo incluye el acceso físico sino también la apropiación simbólica. En otras palabras, la relación binaria entre sujeto y objeto da lugar a la tríada sujeto, objeto e interacción. Para Batista (2014, p.57, traducción nuestra), la apropiación

[...]supone una relación dialéctica en la que el sujeto frente al objeto desarrolla habilidades para construir sus representaciones del mundo, es decir, construcciones simbólicas, permitiendo que el objeto adquiera significados que expresan y producen la subjetividad del sujeto.

Según indican Santos et al. (2017), la cuestión del acceso a la información impacta significativamente en el campo de la Ciencia de la Información. De este modose enfatiza la necesidad de reflexionar desde este campo sobre la aplicabilidad de la LAI en el contexto social de todos los ciudadanos.Esta necesidad también abre nuevas posibilidades paraconsiderar los estudios de la información en el ámbito científico y así comprender los fenómenos sociales, principalmente cuando estos 
fenómenos tienen relación con las innovaciones tecnológicas que posibilitan y requieren nuevos modos de relacionarse con los otros y con los aparatos tecnológicos. Sousa (2012, p.72, traducción nuestra) señalaal respecto de la relación entre usuario y máquina que "con la creciente complejidad computacional de los métodos de interacción, el foco se puso en la relación humano-ordenador, además de en el desempeño del usuario". Lemos (2005, p.2, traducción nuestra)también presenta su visión acerca de este asunto: "Las tecnologías digitales ylas nuevas formas de conexión inalámbrica promueven usos flexibles del espacio urbano [...]". Para el autor:

Estamos en la era de la conexión. Esta no essolola era de la expansión de los contactos en forma de relaciones telemática [...] Se trata de la ampliación de formas de conexión entre hombres y hombres, máquinas yhombres, y máquinas y máquinas motivadas por el nomadismo tecnológico de la cultura contemporánea [...] (LEMOS, 2005, p.2, traducción nuestra).

Este aspecto ha sido relacionado en el campo de la Ciencia de la Información (CI) con conceptos como el de alfabetización informática y la brecha digital. Sobre el concepto de brecha digital, Richard Stallman (2009), afirmaba que un uso incorrecto de las Tecnologías de la Información y la Comunicación(TIC) podría ser incluso peor que su inexistencia: "La idea de la brecha digital presupone que la cuestión es tener o no tener computadora. No estoy de acuerdo, porque tener acceso a las computadoras no es bueno si el software te priva de la libertad. Windows y Macintosh son propietarios. Llevar a más gente a usar Windows o Macintosh no es un avance social. La distinción principal es usar software propietario o evitarlo". De manera análoga,podría indicarse que la cuestión principal del acceso a la información no es hacer toda la información pública disponible digitalmente, o utilizar tecnologías propietarias o complejas para su tratamiento y acceso, sino asegurarse de una efectiva e inclusiva apropiación de la información por parte de la población.

Jesús González Barahona (2002b) analizó la relación existente entre la imparcialidad de los estados y el funcionamiento de la industria del software. En este trabajo, Barahona cuestiona la imparcialidad de los estados indicando que se priman unos modelos de negocio sobre otros, principalmentea través de la legislación pertinente en materia de propiedad intelectual y propiedad industrial, aunque también afectados por otros aspectos. Un sistema público que privilegie unos modelos tecnológicos y de acceso a la información éticos obtendría como resultado beneficios económicos para la administración pública, asícomo un 
concepto de libertad más realizado para la población (MARTÍNEZ-ÁVILA; PRIETO-GORDO, 2009).

Para Antonio García Gutiérrez (2016), la imposición de tecnologías digitales sobre la población sería una nueva forma de totalitarismo, una nueva ofensiva digital del capitalismo. Según Sousa (2012), aunque la información adquiere un nuevorol social y económicoen la actual sociedad de la información, los profesionales de la información necesitan afrontar los nuevos desafíos que surgen con cada innovación. Shapiro y Hughes (1996) presentan el concepto de alfabetización informática en función de un programa basado en otras siete alfabetizaciones: alfabetización en herramientas (conocimiento y uso de las herramientas dentro de las Tecnologías de la Información, incluyendo el hardware y el software); alfabetización en recursos (conocimiento de los sistemas de acceso a los recursos de información); alfabetización socio-estructural (comprensión de la situación social y de producción de la información); alfabetización investigadora (uso de las herramientas de las Tecnologías de la Información aplicadas a la investigación y el trabajo académico); alfabetización para la publicación (habilidad para difundir y publicar información); alfabetización en las tecnologías incipientes (capacidad para comprender las innovaciones en Tecnologías de la Información y la toma de decisiones con respecto a las nuevas tecnologías); y alfabetización crítica (capacidad para evaluar de forma crítica los beneficios y costes de las tecnologías de la información). Sousa (2012) señala que hay una gran dificultad en convertir la información en comprensión. Para el autor:

El proceso de desarrollo no se puede guiar solamente por las necesidades impuestas por el mercado, sino también por los aspectos relacionados con los usuarios [...] y tambiénseñala que [...] una cantidad más grande de información,que debería representar más oportunidades para una comprensión del mundo, puede crearinnumerables barreras para el acceso, principalmente porque nosiempre la información que está disponible responde a la necesidad de los usuarios (SOUSA, 2012, p.68, traducción nuestra).

La información es un concepto complejo, y, en el campo de la Ciencia de la Información,se hace necesario destacar que "la cuestión importante no es soloqué significado le damos al término en la CI, sino también cómo se relaciona con otros términos básicos como documentos, textos y conocimiento" (CAPURRO;HJØRLAND, 2003, p.345, traducción nuestra). Teniendo en cuenta los maticesque comprenden la información desde un punto de vista más abstracto y social, destacamos, fundamentalmente, el paradigma social de Capurro. La importancia queCapurro otorga alos estudios del proceso de construcción de la información radica en queel 
autor reconoce la existencia de tres paradigmas en el área: el primero es el denominado paradigma físico; el segundoes identificado como paradigma cognitivo; y el tercero, que es en el que él mismo se sitúa, es denominado paradigma social (ARAÚJO, 2009). Además de Capurro, hay otros autores queenfatizan la incidencia de los factores sociales relacionados con la información (por ejemplo, el propio Hjørlanden su paradigma de análisis de dominio). No obstante, el posicionamiento de Capurro es uno de los más influyentes y citados en la Ciencia de la Información, especialmente en los estudios teóricos. Según el autor, "es relativamente fácil contar el número de palabras en un documento o describirlo de otras formas; lo que es mucho más difícil es intentar descubrir para quién ese documento tiene relevancia y cuáles son las preguntas importantes que ese documento puede responder" (CAPURRO; HJØRLAND, 2003, p.397, traducción nuestra).

Pese a que elparadigmasocial de Capurroresulta de gran relevancia eimpacto en la $\mathrm{CI}$, su aplicación aún sigue siendo complejayaque no existen demasiadosestudios que pongan en práctica esta propuesta para la comprensión de la información científica. Aunque el acceso a la información sea un fenómeno semejante en diferentes contextos, los modos de realizar las apropiaciones por diferentes sujetos son diversos y las comprensiones y apropiaciones diferentes. Este sería el caso del acceso a la información para el contexto electrónico,en el que además de la información disponible entran en juego, entreotros, aspectos relacionados con la accesibilidad y usabilidad de las tecnologías. En este contexto, y considerando la gran difusión y ubicuidad de las TIC, también resulta pertinente el concepto de la Arquitectura de la Información (AI) en relación a los problemas del acceso a la información pública. Sousa (2012) destaca que en el escenario tecnológico actual:

[...] existe la necesidad inmediata de revisión de los sitios electrónicos o websites, considerando los principios de la Arquitectura de la Información (AI), con las recomendaciones sugeridas para alcanzar Usabilidad y Accesibilidad, ya que no se pueden ignorar laslimitaciones físicas o cognitivas de los usuarios que existen o existirán en el futuro (SOUSA, 2012, p.67, traducción nuestra).

En este sentido, resaltamos que el mero acceso a la información no es suficiente para satisfacer al usuario. Cruz (2013, p.379, traducción nuestra) señala que "el reconocimiento de la legitimidad del derecho a la información solo se realizará con el aprendizaje y la internalización de su concepto". Para Sousa (2012) los ciudadanos necesitan comprender de un modoadecuado la información disponible, es decir, resulta fundamental 
que se consideren las cuestiones cognitivas del usuario. En otras palabras, como los websitesno siempre consiguen ofrecer la información de un modo apropiado, los usuarios tampoco consiguen encontrar la información de una manera satisfactoria,entre otras cosas también porque no existió una representación adecuada previa. Este aspecto tendría también relación con el concepto de alfabetización tecnológica, que según establece Richard W. Budd (1997, no paginado), consiste en

la habilidad para buscar, encontrar, ordenar, categorizar y organizar información para el uso personal y profesional. Cómo conseguir el acceso y el uso de Internet y otros bancos de datos en línea relevantes.

En este sentido también cabe destacar la necesidad de adaptación y transformación en el mundo digital, ya que "la introducción de las TIC en la esfera de la Administración Pública ha implicado notables cambios en la gestión de sus recursos de información y permite que la información sea procesada y compartida de un modo diferente" (MARTÍNEZ et. al, 2018, p.179). Al destacar la actuación del usuario, los autoresafirman que: "[...] tanto ciudadanos como profesionales de la información y de la documentación deben adaptarse a los nuevos cambios, ya que las TIC implican una importante transformación en el modo de comunicarse con los organismos públicos" (MARTÍNEZ et. al, 2018, p.180).

\section{La Ley de Acceso a la Información en Brasil}

La elaboración de leyes requiere varios trámites de acción que resultan en un conjunto de procedimientos previamente establecidos por los Parlamentarios, los cuales tienen la función de legislar y fiscalizar. Estos trámites se realizan según el reglamento interno y reciben el nombre de proceso legislativo. Este proceso se inicia con la presentación de un proyecto de ley el cual pasa por diversas etapas de análisis y votación. La LAI en Brasil fue aprobada a los dieciocho días del mes de noviembre del año 2011, tras numerosas discusiones iniciadas en el año de 2005.

Figura 1 - Trayectoria de la LAI 


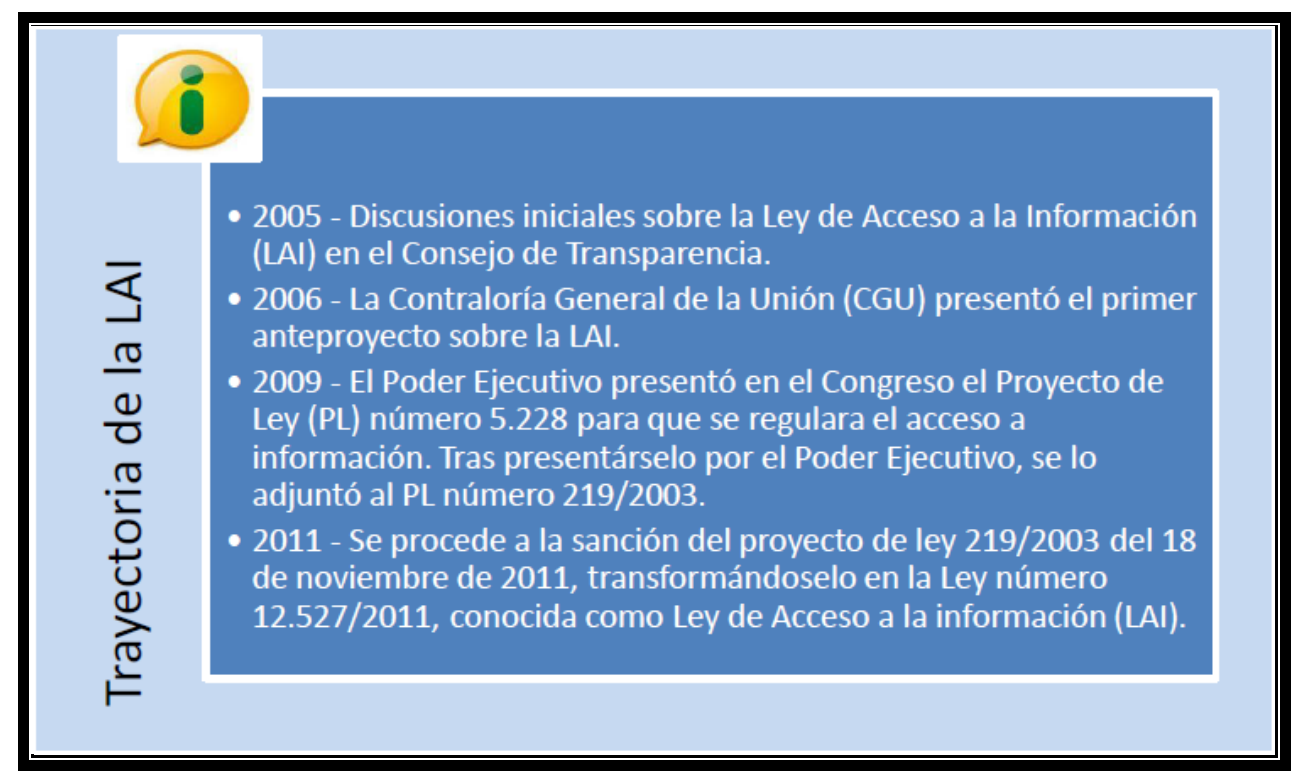

Fuente: Elaboración propia

La LAI busca regular el inciso XXXIII del artículo $5^{\circ}$ del I Capítulo de los Derechos y Obligaciones Individuales y Colectivos de la Constitución Federal - y establece que:

El artículo $5^{\circ}$ de la Constitución Federal de Brasil de 1988 es uno de los más importantes del texto, por tratar de principios relativos a los derechos y garantías fundamentales de la Carta Magna de 1988(BRASIL, 2016). Todos estos principios consagran la primacía de los derechos fundamentales, recuperados y ampliados tras 20 años de dictadura militar en los que muchos habían sido suprimidos, incluyendo la libertad de expresión y el acceso a la información. De hecho,

[...] la LAI en Brasil fue también posible por las reivindicaciones del derecho a la verdad y a la memoria. La LAI conto con una fuerte participación de la sociedad civilorganizada, como periodistas, familiares de las víctimas de políticos y académicos desaparecidos, así como también contó con una legitimación de las decisiones tomadas por la Corte Americana de Derechos Humanos y del Judicial nacional (SOUSA, 2012, p.67, traducción nuestra).

Los derechos fundamentales, en líneas generales, son los derechos positivados,es decir, aquellos que forman parte de los derechos de un ordenamiento jurídico, lo que implica la posibilidad de hacerlos efectivos materialmente perdiendo su carácter únicamente idealista. Los derechos fundamentales son todos aquellos que confieren dignidad a las personas, inherentes a ellas por el sólo hecho de ser personas. 
Los derechos fundamentales son derechos individuales o colectivos. Los derechos individuales, también llamados derechos civiles, se refieren a la vida, la igualdad, la libertad, la seguridad y la propiedad de los individuos, garantizandosu autonomía frente a la sociedad. En lo que respecta a los derechos colectivos, aunque resulta difícil establecer una definición exacta, puede afirmarse que son todos aquellos que van más allá de la esfera estrictamente individual y que proceden de logros sociales. La mayoría de los derechos colectivos forman parte de los derechos sociales.

La Constitución Federal de 1988 trata en su artículo $5^{\circ}$ de los derechos individuales, de los colectivos, y delos deberes. Los deberes que derivan del artículo50 y sus incisos se destinan, principalmente, al Poder Público y sus agentes, los cuales no pueden violarlos por corresponder a estos agentes públicosel deber de dar un buen ejemplo a la sociedad. El derecho a la información es un derecho fundamental y colectivo, el cual se refiere al derecho colectivo o de la colectividad a la información (SILVA, 2005).

Aunque el derecho de informar, en un principio, podría parecer un derecho individual, debido a la libertad de manifestación de pensamiento, su sentido colectivo resultamucho más fuerteactualmente debido a los grandes cambios quehan sufridolos medios de comunicación de masas en los últimos tiempos. De esta forma, su actual sentido supera el antiguo derecho de libertad de prensa que constituía el derecho subjetivo de manifestación de pensamiento de una persona, derecho que derivaba de la libertad individual. Por otro lado, tanto el derecho fundamental como el derecho colectivo son tratadosen la Carta Magna de 1988. Silva (2005, p.260), al abordar la temática en el capítulo de la comunicación (art. 220 al 224), señala que "[...] la libertad de información debe dejar de ser una simple función individual para convertirse en una función social".

Partiendo de estos principios, el artículo $5^{\circ}$, en su párrafo $1^{\circ}$, de la Constitución Federal de 1988, establece que las normas que definen los derechos y garantías fundamentales tienen aplicación inmediata(BRASIL, 2016). Este dispositivo constitucional evidencia el poder normativo del que gozan los derechos fundamentales, como consecuencia de la relevancia que el poder constituyente originario otorgó a todos ellos sin hacer ninguna distinción entre sus categorías y con el objeto de positivarlos en el texto constitucional buscando su real eficacia.

Los problemas y necesidades abordados por la LAI vienen siendo discutidos desde los años 40 con la Declaración de Derechos Humanos de 1948. Estas preocupaciones han sido reflejadastambién más recientemente en la Constitución Federativa de Brasil de 1988 comoen continuas discusiones y declaraciones de importantes órganos de la comunidad internacional como la Organización de las Naciones Unidas 
(ONU) y la Organización de los Estados Americanos (OEA). Según declaraciones de la OEA, el objeto de la LAI también estaría presente en la Convención Americana de Derechos Humanos de 1969, en la Carta Democrática Internacional de 2001 y en la Declaración de la Cumbre de las Américas de Nuevo León de 2004, según se puede observar en la Tabla 1.

Tabla 1:Presencia del objeto de la LAI en documentos internacionales

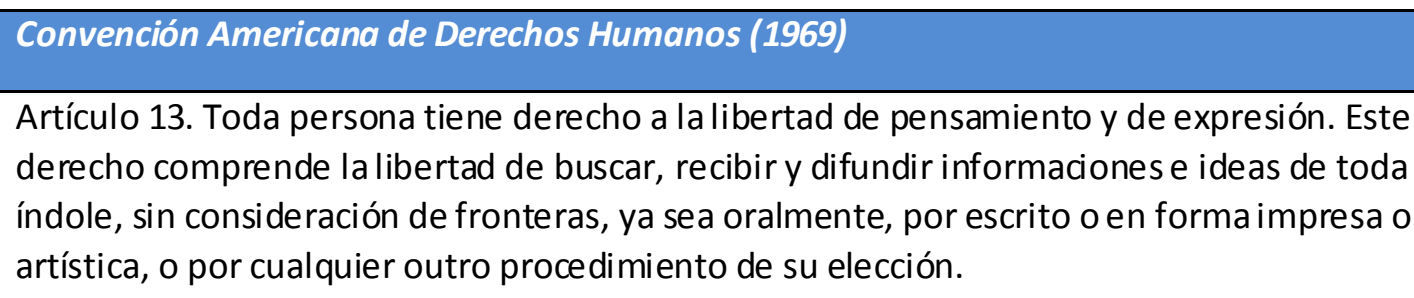

Artículo 3. "Los Estados Partes convienen em considerar la aplicabilidad de medidas, dentro de SUS propios sistemas institucionales, destinadas a crear, mantener y fortalecer normas de conducta para el correcto, honorable y adecuado cumplimiento de las funciones públ icas y Mecanismos para hacer efectivo el cumplimiento de dichas normas de conducta."

Declaración de la Cumbre de las Américas de Nuevo León (2004)

"El acceso a la información em poder del Estado, com el debido respeto a las normas constitucionales y legales, incluídas las de privacidad y confidencialidad, es condición indispensable para la participación ciudadana y promueve el respeto efectivo de los derechos humanos."

Fuente: <http://www.oas.org/es/sap/dgpe/acceso/mandatos.asp>. Accesoen 01 mar. 2019.

Las discusiones sobre el objeto de la LAI están presentes en numerosos países. Según los datos de la OAE existen 22 países de América que en el año 2015 contaban con una ley que consagra el derecho de acceso a la información pública (Figura 2). 
Las limitaciones de la Ley de Acceso a la Información en Brasil: reflexiones teóricas en el ámbito de la ciencia de la información
Ellen Vallota Elias Borges; Daniel MartínezÁvila; Mariana Rodrigues Gomes de Mello

Figura 2: Las leyes de acceso a la información en los países de América

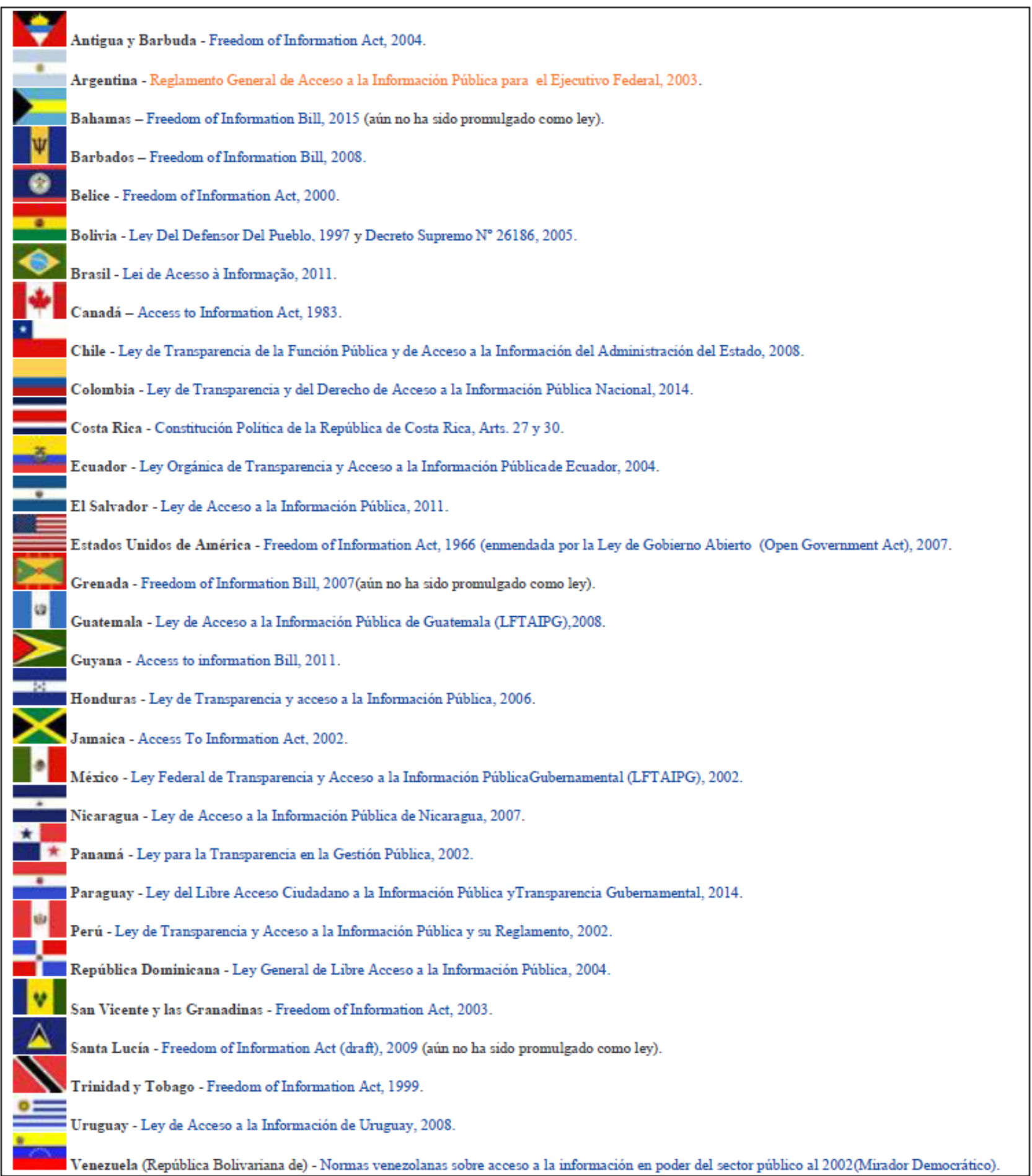

Fuente:<http://www.oas.org/es/sap/dgpe/acceso/mandatos.asp>. Acceso en: 01mar. 2019.

\section{Las tecnologías y el contexto de la LAI}

Las evoluciones tecnológicas y económicas están estrechamente ligadas alas relaciones entre los países y sus leyes. Este aspecto a su vezpropiciagrandes reflexiones acerca de la aplicabilidad, accesibilidad y usabilidad de las informaciones públicas en cada país. Para Sousa: 
[...] aunque se consideren los principios de la AI, también existe la necesidad de considerar aspectos relacionados con la usabilidad y accesibilidad, ya que un websitepodría seguir los referidos principios y no ser simpleen la utilización y acceso a la información (SOUSA, 2012, p.72, traducción nuestra).

Estudiar la LAI bajo una perspectiva práctica que busque comprender su aplicabilidad requiere una comprensión de las relaciones de las condiciones de acceso con las condiciones del usuario. En otras palabras, la LAI está estrechamente relacionada con el ciudadano y sus condiciones de acceso a la información. Sobre esta perspectiva, queda claro que, aunque el acceso garantiza la posesión de los documentos, no garantiza de forma efectiva la apropiación de la información plasmada en estos documentos.

Las nuevas tecnologías y medios de comunicación producen una nueva realidad social que promueve diversas relaciones sociales, incluso en el contexto político. En un reciente estudio sobre la actuación de las redes sociales se comprobó empíricamente que:

[...] asistimos a un robustecimiento de las redes sociales como una herramienta útil para la información política. Su relevancia hace que sean uno de los principales vehículos para que los líderes puedan enviar sus mensajes políticos a los potenciales electores (GONZÁLEZ; CAMBRA, 2018, p.189).

Aunque la tecnología no actúe directamente, forma parte de todo el contexto social actual. Los viejos medios y los nuevos medios están estrechamente relacionados enlo que Jenkins llamade convergencia mediática. Para el autor:

La convergencia mediática es más que un mero cambio tecnoló gico. La convergencia altera la relación entre las tecnologías existentes, las industrias, los mercados, los géneros y el público. La convergencia latera la lógica con la que operan las industrias mediáticas y con la que procesan la información y el entretenimiento los consumidores de los medios. Tengan bien presente que la convergencia se refiere a un proceso, no a un punto final (JENKINS, 2008, p.26).

Ante lo expuesto, secomprende la necesidad de entender las nuevas relaciones y la actuación de los nuevos medios de comunicación y tecnologías. Para ello, en 2010 se creó en Brasil la Cartilla de Usabilidad(BRASIL, 2010), en la que se presenta el concepto de 
usabilidad, sus objetivos y las directrices de usabilidad parael Gobierno electrónico. Según se indica en la Cartilla, estas directrices son orientaciones que deben considerarse en el desarrollo de un sitio web. Las directrices dela Cartilla se centran en las necesidades delos ciudadanos y se basan en los problemas comunes encontrados en las páginas web de las instituciones públicas de Brasil. Lasdirectrices son (BRASIL, 2010):

- Directriz 1: contexto y navegación en internet

- Directriz 2: carga de información

- Directriz 3: autonomía

- Directriz 4: errores

- Directriz 5: diseño

- Directriz 6: redacción

- Directriz 7: consistencia y familiaridad

En lo relativo ala redacción, debe señalarse que éste es uno de los principales aspectos en los que se centra la presente investigación, ya quetrabaja con la premisa de que el acceso no sirve de nada si no hay una comprensión efectiva de las informaciones disponibles (apropiación). Según la Cartilla, los sitios web del gobierno tienen la función de prestar servicios a los ciudadanos, de esta manera, deben tenerse en cuenta las características delos usuarios y su conocimiento: "El sitio web debe hablar la lengua de las personas, con palabras, frases y conceptos familiares además del texto ser objetivo" (BRASIL, 2010, p. 26, traducción nuestra).

En 2011 se creó en Brasil laCartilla de Acceso a la Información Pública, impulsada por la Contraloría General de la Unión(CGU) en colaboración con la Organización de la Naciones Unidas para la Educación, la Ciencia y la Cultura - UNESCO. Esta Cartilla buscaba una mejor comprensión de los principales aspectos de la Ley según los siguientes puntos:

a) Con la aprobación de la LAI, Brasil da un paso significativo hacia la consolidación de su régimen democrático, incrementando la participación ciudadana y fortaleciendo los instrumentos de control de la gestión pública;

b) La Ley no sólo garantiza al ciudadano el derecho de acceso a la información, sino también el cumplimiento del compromiso contraído por el país ante la comunidad internacional en varios tratados y convenciones;

c) La Ley 12.527 representa un cambio de paradigma respecto a la transparencia pública ya que establece que el acceso a la información es la regla y el sigilo es la excepción. Aunque todos los ciudadanos pueden solicitar acceso a la información pública el retose centra en asegurar la aplicación de la Ley. Se hace necesario hacer frente a una serie de desafíosde naturaleza técnica, tecnológica y 
administrativa que requieren recursos financieros $y$ humanos (debidamente capacitados) para garantizar el cumplimiento de la Ley.

Ante todo,se desprende que la responsabilidad de garantizar la transparencia y el acceso a la información pública por parte de los agentes públicos es algo esencial para que se cumpla la ley de un modo eficaz.

Como ya ha sido expuesto anteriormente, el acceso a la información es un derecho fundamental establecido en el ordenamiento jurídico brasileño, en el artículo $5^{\circ}$ inciso XXXIII,en el inciso II del $\S 3$ del art. 37 y en el $\S 2$ del artículo 216 de la Constitución Federal de 1988, que determina que: "Corresponde a la administración pública, según la ley, la gestión de la documentación gubernamental y la adopción de las medidas necesarias para su consulta por todos los que la necesitan" (BRASIL, 1988, traducción nuestra). Este dispositivo constitucional se complementa por la Ley Federal 8.159, que trata de la política nacional sobre los archivos públicos y privados, y que requiere una gestión documental eficiente, protección de los archivos (art.10) y garantía de acceso a las informaciones gubernamentales por cualquier ciudadano (art. $4^{0}$ y 22). Por lo tanto, la Ley $12.527 / 2011$ (LAI) ratifica un compromiso que ya había sido firmado a nivel constitucional.

Por otro lado, acontecimientos recientes y fuerzas políticas gubernamentales antidemocráticas han abogado por limitar el acceso a la información pública, movidos por intereses que privilegian el obscurantismo político y la falta de transparencia enlas actividades de la administración pública. Esta oposición a la transparencia y acceso a la información se ha visto motivadapor una oposición al avance de los derechos de la población y su capacidad para fiscalizar la gestión de los recursos públicos. El decreto anticonstitucional 9.680, que limita el acceso a la información, sancionado por el Vicepresidente de la República y publicado al comienzo del año 2019, no solo indica un retroceso legal significativo en relación al acceso a la información, sino también un retroceso ético y educativo al evidenciar una oposición del gobierno actual a una apropiación de la información legítima por parte de la población, la cual produciríatransformaciones sociales y construcción de nuevos conocimientos. El 19 de enero de 2019, la Cámara de los Diputados Federales votó, con carácter de urgencia, un proyecto que detuviera los efectos del decreto que ha modificado las reglas de la LAI. Sin embargo, el texto aún tendría que pasar por el Senado para su aprobación. Frente a esta situación, el 26 de febrero de 2019,el presidente de Brasil Jair Bolsonaro derogó el decreto firmado por el presidente en ejercicio, Hamilton Mourão, que delegaba a los empleados comisionados la definición de sigilo de datos (SIMÕES, 2019). 


\section{Objetivos y metodología}

El trabajo presenta los siguientes objetivos:

a) Comprender cómo se reconoce la difusión de la LAI en el Portal del Gobierno Federal de Brasil así comola presencia delas autoridades competentes;

b) Verificar el número de autoridades presentes y su disponibilidad para contacto;

c) Verificar la actuación de las autoridades encargadas de ejecutar la ley dentro del Sistema electrónico de atención al ciudadano (e-SIC);

d) Analizar la aplicabilidad de la LAI en función de los servicios disponibles para solicitarinformacióna travésdel portal del Gobierno Federal;

e) Comparar los puntos de convergencia y divergencia en las respuestas a las solicitudes de información enviadas por las autoridades competentes.

El objeto principal de este trabajo es el proceso de apropiación de la información en relación con la aplicabilidad de la ley,a través de las solicitudes de información realizadas en el portal del Gobierno Federal de Brasil. De este modo, se introduce un matiz diferencial con respecto a otros trabajos existentes en el campo de laCI, en relación con la apropiación práctica de la información.En este sentido, el presente trabajo pone de relevancia la necesidad de abordar el objeto información en un contexto interdisciplinar, en conjunción con el derecho, buscando poner en práctica el análisis delos procesos de producción y apropiación de la información. El estudio también busca explorar los resultados presentados por las autoridades responsables por la ejecución de la LAI y su interacción con el entorno social, proporcionando fundamentos para el análisis. Para comprender este propósito se parte dela distinción teórica realizada por Almeida Júnior (2013) en relación a la diferencia entre información y documento. Para este autor,la información no es algo que puede almacenarse, sino que sonlos documentos los que se almacenan, aglutinan o depositan en la biblioteca. En este sentido, puede decirse que la conversión de documentos en información depende de la mediación (ALMEIDA JÚNIOR, 2013). En este sentido, se buscó verificar la opinión de las autoridades competentes en relación al contenido de la LAI en lo que se refiere a la relación entre acceso y apropiación. Para ello, tras localizar la lista de todos los SICs (Sistemas de atención al Ciudadano) disponibles en el portal del Gobierno Federal de Brasil ${ }^{1}$, en los que se presentan los nombres y apellidos, teléfonos y direcciones de correo electrónico de las

\footnotetext{
${ }^{1}$ SICs: lista de SICs. Disponível em: <http://www .acessoainformacao.gov.br/lai-para-sic/sic-apoioorientacoes/lista-de-sics >. Acesso em: 10 out. 2017.
} 
autoridades competentes de ejecutar y supervisar la LAI, se pudo realizar un primer contacto con estas autoridades mediante correo electrónico.

Tras las indicaciones de las primeras respuestas de algunas autoridades, se modificó la estrategia de contacto y obtención de información. Tal como fue indicado, se realizó un registro en el e-SICpara contactar con las autoridades registradas mediante una solicitud registrada en el sistema. Esta acción generó un número de solicitud encaminadopor el sistema al órgano competente y en el que incluyóel plazo para la devolución de la respuesta, tal como es establecido por la Ley. De este modo, pudo desarrollarse el estudio utilizando una metodología que comprendió las siguientes etapas:

a) Investigación y lecturas. Para comprender la actuación de las autoridades responsables por la aplicación de la LAI se verificó el modo de difusión de la Ley a través del portal del Gobierno Federal, disponible en la dirección http://www.acessoainformacao.gov.br/. En un segundo momento también se realizó una lectura profunda tanto de la estructura y contenidos de la LAI comode los aspectos disponibles en el portal, con el objetivo de reflexionar y elaborar un cuestionario interdisciplinar para investigar la aplicabilidad de la LAI en relación a los conceptos de acceso y apropiación de la información (tal como son estudiados en la $\mathrm{CI}$ ).

b) Acceso al portal del Gobierno Federal. Tras la lectura y comprensión de la información del portal se realizó un análisis de los datos disponibles, sistematizándolos en una lista con el nombre, apellidos, teléfonos, dirección y correo electrónico de las autoridades competentes. En esta etapa también se realizó un primer contacto por email con estas autoridades responsables por la aplicación de la LAI.

c) Acceso al portal electrónico (e-SIC). Tras contactar por correo electrónico con las primeras autoridades de la lista, se realizó una reformulación de la estrategia de obtención de datos en función de las nuevas orientaciones recibidas. De forma análoga a la experiencia de un ciudadano modelo (utilizando la terminología de Umberto Eco, 1993) en el proceso de acceso a la información, fuimos informados que el contacto mediante correo electrónico no sería el canal más adecuado para realizar la solicitud, por lo que se procedió al contacto con las autoridades responsables a través del e-SIC ${ }^{2}$.Tras comparar la cantidad de órganos presentes en la lista de los SICs y los órganos registrados en el e-SIC, se envió un cuestionario formado por 10 preguntas relacionadas con la aplicabilidad de la LAI y el acceso y apropiación de la información a los 323 órganos registrados en este sistema.

\footnotetext{
${ }^{2}$ E-SIC: sistema eletrônico do serviço de informação ao cidadão. Disponível em: <https://esic.cgu.gov.br/sistema/site/index.aspx>. Acesso em: 15 set. 2017.
} 
d) Análisis de los datos. Se realizó un análisis detallado de las respuestas obtenidas de los órganos competentes y se reorganizó la cantidad de órganos a ser evaluados en un atabla. Se verificó la aplicabilidad de la LAI según el análisis de las respuestas obtenidasde las autoridades competentes y se contactaron los órganos pertinentes cuando existieron divergencias en relación al número de organismos registrados.

El proceso de búsqueda de información y contacto con las autoridades se realizó durante el segundo semestre de 2017, mientras que las respuestas se obtuvieron en el período comprendido entre noviembre de 2017 y enero de 2018. La validación de los resultados del cuestionario se justifica ya que los datos obtenidos permitieron acceder y analizar la aplicabilidad de la LAI segúnla obligación legal de las propias autoridades de aplicación, encargadas de vigilar la Ley en el ámbito del eSIC.El contacto con las autoridades competentes a través del sistema aseguró una tasa de respuestas satisfactoria, aunque sólo fuera para transmitir una negativa, ya que la CGU está obligadaa garantizar la aplicación de la ley según lo dispuesto en el Decreto no 7.724. En este Decreto se indica que esta forma de supervisión es una forma de garantizar la aplicación de la LAI en el Gobierno Federal, incluyendo actividades de orientación para los órganos y organismos públicos, la difusión de la Ley para la sociedad yla supervisióndel cumplimiento de los procedimientos y plazos establecidos. Basándose en este principio de supervisión de la Ley, se pudo realizar un análisis comparativo entre lo que es establecido por la ley y lo que se hace en la práctica.

Por otro lado, también existió un número de autoridades que no respondieron el cuestionario. Entre las diferentes justificaciones alegadas para noresponderlo la gran mayoría aludió la falta de competencia para realizarlo. En este sentido, el cuestionario fue redirigido por muchas autoridades hacia otros órganos administrativos, principalmentela CGU. Los principales resultados alcanzados en estas etapas permitieron una reflexión sobre los conceptos de acceso y apropiación de la información en el ámbito social de la LAI, tal como ocurriría por ciudadanos modelo. Las preguntas incluidas enel cuestionario están desarrolladas en la Tabla 2. 


\section{Tabla 2: Preguntas elaboradas para la composición del cuestionario} sobre la LAI

\begin{tabular}{|c|c|}
\hline 1 & $\begin{array}{l}\text { ¿La Ley de Acceso a Información (LAI) garantiza el derecho de apropiación de la } \\
\text { información? }\end{array}$ \\
\hline 2 & $\begin{array}{l}\text { Con la aprobación de la ley } 12.527 \text {, de } 18 \text { de noviembre de } 2011 \text {, la Ley de Acceso a } \\
\text { Información, Brasil da un paso adelante para la consolidación de la democracia, lo que } \\
\text { amplía la participación de todos los ciudadanos, fortaleciendo los instrumentos de control } \\
\text { y gestión pública. ¿Es posible la consolidación de un régimen democrático sin que la } \\
\text { apropiación de la información se integre al acceso previsto en la LAI? }\end{array}$ \\
\hline 3 & $\begin{array}{l}\text { Según la LAI, todo ciudadano puede solicitar acceso a informaciones públicas. ¿Es } \\
\text { posible decir que todo ciudadano también puede apropiarse de forma efectiva de las } \\
\text { informaciones que están en los documentos? }\end{array}$ \\
\hline 4 & $\begin{array}{l}\text { Según el Manual de Acceso a informaciones públicas elaborado por la Contraloría - } \\
\text { General de la Unión (CGU) en } 2011 \text {, el término información se define del siguiente modo: } \\
\text { "datos procesados o no, que pueden ser utilizados para la producción y transmisión de } \\
\text { conocimiento, presentes en cualquier medio, soporte o formato". Partiendo de la } \\
\text { definición presentada, los datos (procesados o no) son capaces de producir } \\
\text { conocimiento. En este sentido, ¿Se puede decir que el procesamiento de datos es lo } \\
\text { mismo que el proceso de apropiación de la información? }\end{array}$ \\
\hline 5 & $\begin{array}{l}\text { Basándose en los estudios de la Ciencia de la Información (Cl), los términos "Acceso" y } \\
\text { "Apropiación" son esenciales para las discusiones y reflexiones teóricas en el campo } \\
\text { científico de la Cl. Sin embargo, el término apropiación no forma parte de la elaboración } \\
\text { del Manual de Acceso a Informaciones Públicas elaborado por la Contraloría-General de } \\
\text { la Unión (CGU) en } 2011 \text {. De ahí, ¿Se puede señalar que a partir de las concepciones de } \\
\text { acceso a informaciones presentadas en el manual, el acceso es un proceso que supone } \\
\text { la apropiación? }\end{array}$ \\
\hline 6 & $\begin{array}{l}\text { Según el Manual de Acceso a informaciones públicas “ [...] no siempre es posible } \\
\text { garantizar el } 100 \% \text { del acceso, no obstante el administrador tiene la función de fomentar } \\
\text { el esfuerzo de conseguirlo". ¿La LAl establece medidas para fomentar esfuerzos que } \\
\text { contribuy an para la apropiación de la información? }\end{array}$ \\
\hline 7 & $\begin{array}{l}\text { El lenguaje ciudadano es uno de los temas tratados en el Manual de Acceso a } \\
\text { Informaciones Públicas y se señala que "El lenguaje debe ser claro y objetivo durante la } \\
\text { comunicación entre la Administración y el ciudadano. Su objetivo es asegurar una lectura } \\
\text { fácil de las informaciones y de los datos". ¿Es posible lograr esto centrando la atención } \\
\text { solamente en el acceso, excluyendo la necesidad de reflejar sobre la apropiación? }\end{array}$ \\
\hline 8 & $\begin{array}{l}\text { La LAI establece el nombramiento de una autoridad en cada órgano de la administración } \\
\text { encargada de supervisar la aplicación de las políticas establecidas. Una de sus } \\
\text { obligaciones es garantizar la efectividad del derecho de acceso. Según el Manual de } \\
\text { Acceso a informaciones públicas elaborado por la Contraloría -General de la Unión (CGU) } \\
\text { ¿Sería interesante repensar en lo que se refiere a la aplicabilidad de la ley y centrar la } \\
\text { atención no solo en la efectividad del acceso sino también de la apropiación? }\end{array}$ \\
\hline 9 & $\begin{array}{l}\text { ¿Hay un entendimiento homogéneo entre las autoridades encargadas de la ejecución de } \\
\text { la LAl en lo que se refiere a las diferencias entre los términos "documento", "datos" e } \\
\text { "información" presentados en el Manual de Acceso a Informaciones Públicas, basándose }\end{array}$ \\
\hline
\end{tabular}




\begin{tabular}{|l|l|}
\hline & en el área de la Ciencia de la Información (CI)? \\
\hline 10 & $\begin{array}{l}\text { Teniendo en cuenta que el Acceso a informaciones y la Apropiación de informaciones } \\
\text { son procesos diferentes debatidos y reflejados dentro de la Cl, cabe destacar que el } \\
\text { derecho de acceso no garantiza el derecho de la apropiación. De ahí, sería esencial } \\
\text { repensar la LAl basándose en las teorias desarrolladas en el ámbito de la Cl. ¿Se puede } \\
\text { considerar una propuesta viable la integración de investigaciones en el área de la Ciencia } \\
\text { de la Información en las prácticas de desarrollo y aplicabilidad de la LAl? }\end{array}$ \\
\hline
\end{tabular}

Fuente: Elaboración propia.

\section{Resultados y discusión}

La lectura interdisciplinar de la LAI, el acceso a la información presente en el Portal del Gobierno Federal, el contacto con las autoridades competentes y el análisis de las respuestas llevaron a resultados que reflejan no sólola esfera de la elaboración de la Ley sino también de la actuación de las autoridades competentes.

Basándose en las informaciones y las tecnologías ofrecidas por el Portal, se muestra que el mero acceso no es suficiente para lograr una adecuada apropiación de la información. De este modo, fue necesario reflexionar(y hacer reflexionar a las autoridades) sobre las relaciones entre acceso y apropiación en el contexto de la LAI. Se identificaron cómo los dispositivos de mediación ofrecidos por el Estado contribuyen a laconstrucción y comprensión de la informacióndurante los procesos de solicitud.

El uso del e-SIC contribuyea un mejor entendimiento del proceso de solicitudde información a todos los órganos. Sin embargo, durante la recopilación de los datos ofrecidos por el Portal del Gobierno Federal, se detectaron discrepancias entre los datos presentados en la lista completa de los SICsy la cantidad de órganos registrados en el e-SIC. Tras hacer un análisis detallado de estos datos se llegó al número final de 310 órganos para análisis (Tabla 4).

Tabla 4: Cantidad de órganos públicos presentes en el e-SIC y en la lista de los SIC

\begin{tabular}{|l|l|}
\hline \multicolumn{2}{|c|}{$\begin{array}{c}\text { Divergencias de datos relacionados a la cantidad de órganos públicos registrados en la } \\
\text { lista del portal del Gobierno Federal (SIC) y la cantidad de órganos registrados en el } \\
\text { Sistema electrónico de atención al ciudadano (e-SIC) }\end{array}$} \\
\hline 10 Problema & $\begin{array}{l}323 \text { órganos presentes en el e-SIC y } 316 \text { órganos presentes en la lista } \\
\text { completa de los SIC. }\end{array}$ \\
\hline Resultado & 17 órganos del e-sic estaban duplicados, quedando solamente 306 \\
\hline
\end{tabular}




\begin{tabular}{|l|l|}
\hline & órganos para análisis. \\
\hline 2o Problema & $\begin{array}{l}\text { 10 órganos de la lista SICs no estaban presentes en el sistema } \\
\text { electrónico e-SIC. }\end{array}$ \\
\hline Resultado & $\begin{array}{l}\text { Se añadieron los } 10 \text { órganos a los 306 órganos anteriores, resultando en } \\
\text { 316 órganos para análisis. }\end{array}$ \\
\hline 3o Problema & $\begin{array}{l}\text { A lo largo de la organización de los datos, se encontró la duplicación de } \\
6 \text { órganos, convirtiendo los resultados en 310 órganos para análisis }\end{array}$ \\
\hline Resultado Final & $\begin{array}{l}\text { 310 solicitudes de información enviadas a los órganos registrados en el } \\
\text { e-SIC. }\end{array}$ \\
\hline
\end{tabular}

Fuente: Elaboración propia.

De los 310 órganos que recibieron las solicitudes, solamente 145 respondieron los cuestionarios. Las respuestas ofrecidas por las autoridades encargadas de la ejecución de la LAI también presentaron divergencias. En la Tabla 5 se muestran los resultados de las respuestas basándose en la cantidad de respuestas afirmativas y negativas.

Tabla 5: Preguntas elaboradas para los órganos públicos

\begin{tabular}{|c|c|c|c|c|c|c|c|c|}
\hline $\begin{array}{l}\text { NÚMERO DE } \\
\text { LAS }\end{array}$ & \multicolumn{2}{|c|}{$\begin{array}{l}\text { Cantidad de } \\
\text { Respuestas Sí }\end{array}$} & \multicolumn{2}{|c|}{$\begin{array}{c}\text { Cantidad de } \\
\text { Respuestas NO }\end{array}$} & \multicolumn{2}{|c|}{$\begin{array}{c}\text { Cantidad de } \\
\text { Respuestas sí y NO }\end{array}$} & \multicolumn{2}{|c|}{ Sin Respuesta } \\
\hline 1 & 74 & $51 \%$ & 68 & $46,8 \%$ & 1 & $0,6 \%$ & 2 & $1,3 \%$ \\
\hline 2 & 43 & $29,6 \%$ & 98 & $67,5 \%$ & 0 & $0 \%$ & 4 & $2,7 \%$ \\
\hline 3 & 69 & $47,5 \%$ & 73 & $50,3 \%$ & 1 & $0,6 \%$ & 2 & $1,3 \%$ \\
\hline 4 & 26 & $17,9 \%$ & 117 & $80,6 \%$ & 0 & $0 \%$ & 2 & $1,3 \%$ \\
\hline 5 & 75 & $51,7 \%$ & 64 & $44,1 \%$ & 0 & $0 \%$ & 6 & $4,1 \%$ \\
\hline 6 & 93 & $64,1 \%$ & 50 & $34,4 \%$ & 0 & $0 \%$ & 2 & $1,3 \%$ \\
\hline 7 & 65 & $44,8 \%$ & 76 & $52,4 \%$ & 0 & $0 \%$ & 4 & $2,7 \%$ \\
\hline 8 & 96 & $66,2 \%$ & 42 & $28,9 \%$ & 0 & $0 \%$ & 7 & $4,8 \%$ \\
\hline 9 & 83 & $57,2 \%$ & 49 & $33,7 \%$ & 0 & $0 \%$ & 13 & $8,9 \%$ \\
\hline 10 & 106 & $73,1 \%$ & 27 & $18,6 \%$ & 0 & $0 \%$ & 12 & $8,2 \%$ \\
\hline
\end{tabular}

Fuente: Elaboración propia. 
La falta de competencia para responder el cuestionario,alegada por algunas autoridades,corrobora quelos problemas presentados por Damatta(2011) todavía no han sido solucionados en lo que se refiere a la actuación de las autoridades competentes encargadas de efectivar la LAI. Damattaya constataba en 2011 indicabala necesidad de capacitar las autoridades para el tratamiento de la información pública:

[...] latransición para un ambiente de transparencia y amplio acceso requiere la sensibilización y capacitación de las autoridades responsables para que lideren, en sus respectivas áreas y frente a sus equipos, los cambios necesarios para el tratamiento de la información pública en el día a día (DAMATTA, 2011, p.13, traducciónnuestra).

Damattaindicaba este aspecto relacionado con la falta de competencia en el tratamiento de la información por parte los diversos órganos dela Administración Pública Federal en los siguientes términos: "Cuando se les pregunta a los órganos sobre los responsables por atender y contestar las solicitudes de información resulta evidente la falta de una postura sistematizada" (DAMATTA, 2011, p.13, traducción nuestra). Partiendo de lo expuesto por este autor, y teniendo en cuenta los resultados de nuestra investigación, comprobamos que tras más de siete años desde la aprobación de la LAI en Brasil todavía existe la necesidad de ofrecer más cursos y capacitaciones sobre los valores y la cultura de acceso a la información pública en el Poder Ejecutivo Federal brasileño. Además de esto, también se detecta la necesidad de reflexionar sobre la actuación de los profesionales de la información en relación aun mejor desarrollo de la aplicabilidad de la LAI, ya que la gran divergencia de respuestas ofrecidas por las autoridades competentessugierela necesidad de una mayor reflexión sobre la forma en que se garantiza el acceso a la información.Este acceso debe ser pensado de un modo integrado, utilizando tecnologías adecuadas y responsablesy que responda un proceso adecuado de mediación que ofrezca un lenguaje más accesible. Se revela por lo tanto la necesidad de repensar la Ley de un modo más crítico y reflexivo, considerando no sólo el acceso sino también el posterior proceso de apropiación incluso por parte de las autoridades competentes.

\section{Conclusión}

Las solicitudes enviadas a los 310 órganos del Gobierno Federal devolvieron respuestas de 145 órganos del Gobierno. Esta cifra se trata de un número bastante significante que permite delinear un panorama general válido de la aplicabilidad de la LAI a través de las actitudes de las propias autoridades encargadas de supervisar la Ley. El análisis y 
discusión de las respuestas de las autoridades hizo posiblellegar alas siguientes conclusiones:

a) Las deficiencias identificadas en el proceso de acceso y apropiación de la informaciónestán relacionadas tanto con la elaboración de la Ley como con la actuación de las autoridades competentes, las cuales no tienen una comprensión homogénea y necesaria del significado real de su función. Mientras que muchas autoridades respondieron el cuestionario tan pronto como tuvieron acceso, otros organismos no los respondieron aduciendo que el pedido no correspondía con el tipode información que están obligados a proporcionar,o alternativamente alegando una falta de competencia;

b) Teniendo en cuenta que las autoridades registradas en la web son responsables por la ejecución de la LAI, se hace necesario que todas ellas presenten una visión y conocimiento semejante sobre la LAI para que puedan actuar de modo semejante;

c) Respecto a la LAI, debe señalarse que, en los tiempos actuales, y principalmente en países como Brasil, el poder económico se ha reveladocomo un obstáculo inmediato para la aplicación efectiva de los derechos fundamentales relacionados con la información. Esta dimensión de la aplicabilidad de la LAI considera que el acceso a la información, además de ser un derecho fundamental, también actuaría como unaherramientapara luchar conta la desinformación y las fakenews propiciadaspor determinados grupos de poder;

d)Aunque el Estado ofrece diversasopciones de acceso a la información, las barreras sociales que impiden a los individuos realizar una apropiación de la información de forma amplia y reflexiva son aún grandes. De esta manera, el presente estudio ha identificado que el acceso a la información, aunque se trate de un derecho fundamental, presenta problemas para su concretización material. En relación a esto se considera que la apropiación de la información se presentaría comouna opción para aumentar su eficacia social.

Teniendo en cuenta lo expuesto, puede afirmarse que, aunque la LAI no considera la las ventajas de la apropiación de la información en su elaboración, la eficacia social de los servicios ofrecidos continúa dependiendo de un proceso de apropiación no incentivado. Los poderes políticos siguen sin valorar la figura del profesional de la información que con su conocimiento contribuye al desarrollo social y económico de la administración. También hay que sumar la deficiente actuación de muchas autoridades competentes que forman parte de la LAI y que no reconocen o colaboran con investigaciones como la presente que podríanreconocer la importancia de estos organismos y su función social. Este tipo de actitudes supone una desmotivación tanto para los órganos que 
demostraron una actitud positiva como para los investigadores que intentar reivindicar el valor de los servicios y del acceso a la información.

A la vista de estos resultados, se infieren diversas implicaciones de la LAI, especialmente en relación a su aplicabilidad social. De este modo,se considera fundamental revisar la actuación de las autoridades encargadas de ejecutar la LAI y promover de una forma más activa un sistema de incentivos por formación e integración con el campo científico de la CI, impulsando la consolidación de estos profesionales en la carrera investigadora de la Administración y enfatizando la importancia de la apropiación de la información. De igual manera, resulta esencial un mayor fomento de la investigación interdisciplinar entre los campos del Derechoy la CI en relación a aspectos relacionados con la apropiación de la información, lo cual permitiría a las autoridades competentes una mayor seguridad y homogeneidad a la hora de orientar a los ciudadanos respecto a las informaciones públicas $y$, en un nivel académico, determinar las implicaciones prácticas de los estudios.

También,como consecuencia del estudio, puede afirmarse que el acceso libre a la información no es el problema en Brasil, ya queéste no sólo es el principal objetivo de la LAI sino que también existe en diversos instrumentos jurídicos que lo respaldan. Tal como está establecido en el artículo 216, párrafo 20, de la Constitución Federal brasileña, "Es competencia de la administración pública, como dispuesto en la ley, la gestión de la documentación gubernamental y las medidas para garantizar su consulta a todos los que la necesitan" (BRASIL, 1988, traducción nuestra). Este dispositivo constitucional es ratificado por la Ley Federal 8.159, que trata de la política nacional sobre archivos públicos y privados, que requiere una gestión documental eficiente, protección de los archivos (art.10) y garantía de acceso a las informaciones gubernamentales a todos ciudadanos (art. 40 y 22). No obstante, las fuerzas políticas de Brasil, y en especial el gobierno que ha tomado el poder tras las elecciones de 2018, están contradiciendo la Constitución Federal que garantiza el acceso a la información como un derecho fundamental. En este sentido, la actitud del nuevo gobierno también está contradiciendo el papel de la Ley de Acceso a la Información queratifica el compromiso de los órganos de actuar con transparencia en la gestión pública. El decreto 9.680, publicado el 24 de enero de 2019 y firmado por Hamilton Mourão, general y vicepresidente de Brasil, establece que dirigentes máximos del Poder Ejecutivo, de autarquías, de fundaciones públicas, de empresas públicas y de economía mixta (empresas que se construyen con capital social parte público y parte privado) pueden decidir si un documento es reservado, secreto o ultrasecreto. Por lo tanto, este decreto viene a limitar el acceso a la información pública convirtiéndose en inconstitucional.Pese a que un decreto nunca podría estar por encima de una norma 
constitucional, y principalmente cuando esta norma trata de un derecho fundamental, el presente gobierno tiene la convicción de vulnerar este fundamental derecho relacionado con la información y efectivar un retroceso en relación a los derechos de la población.

Frente todo lo dicho anteriormente, cabe reiterar que el aporte científico de la CI en relación a la LAI puede producir buenos resultados tanto para las discusiones políticas sobre el acceso a la información como para el funcionamiento de la administración,siempre y cuando las autoridades competentes estén dispuestas a comprender las diferencias fundamentales entre acceso y apropiación de la información. En este sentido resulta importante diferenciar estos dos términos para una adecuada comprensión dela aplicabilidad de la LAI. En otras palabras,podemos concluir que el derecho al acceso no representa el derecho a la apropiación de la información a la vez que si no hay apropiación tampoco hay información. Este problema es uno de los puntos de la LAI en los que no existe una distinción clara entre el derecho del acceso y el derecho de la información. Resulta evidente la imposibilidad de obtener información si se niega el acceso, sin embargo, el libre acceso no está garantizando la información, tal como establece la ley. Dicho esto, se hace necesario que se desarrolle una apropiación de la información para que ésta tenga sentido para quien la acceda.

\section{Referencias}

ALMEIDA JÚNIOR, O. F. Biblioteca pública: avaliação de serviços. Londrina: Eduel, 2013.

ARAÚJO, C. A. A. Correntes teóricas da ciência da informação. Ciência da Informação, Brasília, v.38, n.3, p.192-204, 2009. Disponível em: http://www.scielo.br/pdf/ci/v38n3/v38n3a13.pdf. Acesso em: 05 out. 2018.

BARROS, D. S.; MEDLEG, G. R. Acesso à informação na região nordeste: balanço da criação da LAI nos estados e o processo de sua regulamentação no Maranhão. Perspectivas em Ciência da Informação, Belo Horizonte, v.23, n.1, p. 2-18, 2018. Disponível em: http://www.scielo.br/pdf/pci/v23n1/1413-9936-pci-23-01-2.pdf. Acesso em: 05 mar. 2019.

BATISTA, C.L. Mediação e apropriação da informação pública: a educação fiscal. 2014. Tese (Doutorado em Ciência da Informação) - Escola de Comunicação e Artes. Departamento de Biblioteconomia e Documentação, Universidade de São Paulo, São Paulo, 2014. 
BRASIL. Constituição (1988). Texto consolidado com as alterações determinadas pelas Emendas Constitucionais de Revisão no 1/92 a 91/2016 e pelo Decreto Legislativo no 186/2008. Brasília, DF: Senado Federal, 2016. Disponível em:

https://www2.senado.leg.br/bdsf/bitstream/handle/id/518231/CF88 Livro EC91 2016.pdf. Acesso em: 01 mar. 2019.

Lei no 12.527, de 18 de novembro de 2011. Regula o acesso a informações previsto no inciso XXXIII do art. 50, no inciso II do §30 do art. 37 e no §2० do art. 216 da Constituição Federal, altera a Lei no 8.112, de 11 de dezembro de 1990, revoga a Lei no 11.111 , de 5 de maio de 2005, e dispositivos da Lei no 8.159, de 8 de janeiro de 1991; e dá outras providências. 2011ª. Diário Oficial da União, Brasília, DF, 18 nov. 2011.

Padrões Web em Governo Eletrônico: Cartilha de Usabilidade. Brasília: Secretaria de Logística e Tecnologia da Informação, 2010. Disponível em: https://www.governodigital.gov.br/documentos-earquivos/e-pwg-usabilidade.pdf. Acesso em: 01 mar. 2019.

Decreto no 7.724, de 16 de maio de 2012. Regulamenta a Lei no 12.527 , de 18 de novembro de 2011, que dispõe sobre o acesso a informações previsto no inciso XXXIII do caput do art. 50, no inciso II do $\S 3^{\circ}$ do art. 37 e no $\S 2^{\circ}$ do art. 216 da Constituição. 2012a. Diário Oficial da União, Brasília, DF, 16 maio 2012.

BUDD, Richard W. Información, interacción, intercomunicación: Tejiendo la red global. El impacto de Internet en el futuro de la educación. ZER Revista de Estudios de Comunicación, V.2, n.2, 1997 Disponívelem: http://www.ehu.eus/ojs/index.php/Zer/article/view/17308/15102.Acessoe m: 3 mar. 2019.

CAPURRO, R.; HJøRLAND, B.The concept of information.In: Annual Review of Information Science and Technology, Ed. B. Cronin, v. 37, Chap. 8, p. 343-411, 2003.

CRUZ, E.B. O direito à informação governamental: questões acerca da positividade e legitimação de um direito fundamental. Liinc em Revista, Rio de Janeiro, v.9. n.2, p.370-382, novembro 2013. Disponível em: http://revista.ibict.br/liinc/article/view/3442/3005. Acesso em: 27 mar. 2018.

DAMATTA, R. Sumário executivo:pesquisa diagnóstico sobre valores, conhecimento cultura de acesso à informação pública no Poder Executivo Federal Brasileiro. Controladoria Geral da União, 2011. Disponível em: http://www.acessoainformacao.gov.br/central-de- 
conteudo/publicacoes/arquivos/pesquisadiagnostico.pdf. Acessoem: 01 mar. 2019.

ECO, UMBERTO. Lector in fabula: la cooperación interpretativa en el texto narrativo. Barcelona: Lumen, 1993.

GARCÍA GUTIÉRREZ, ANTONIO. Frentes digitales: totalitarismo tecnológico y transcultura. Salamanca: Comunicación social, 2016.

GONZÁLEZ BARAHONA, Jesús M. La Imparcialidad De Los Estados y La Industria Del Software. TodoLinux, v. 22, p. 12-13, 2002.

GONZÁLEZ, R.B.; CAMBRA, U.C. Información política y redes sociales en Estados Unidos: de Obama a Trump. Informação \& Sociedade, João Pessoa, v. 28, n.3, p.183-191, set./dez. 2018. Disponível em: http://www.periodicos.ufpb.br/ojs2/index.php/ies/article/view/42534. Aces soem: 10 mar. 2019.

JENKINS, H. La cultura de la convergencia de los medio de comunicación. Barcelona, Buenos Aires, México: Paidós. Traducción de Pablo HernidaLascano, 2008. Disponível em: http://www.semioticafernandez.com.ar/wpcontent/uploads/2008/05/2013 jenkins cultura-de-laconvergencia introduccion.pdf. Acesso em: 01 mar. 2019.

LEMOS, A. Cibercultura e mobilidade: a era da conexão. Intercom Sociedade Brasileira de Estudos Interdisciplinares da Comunicação, 2005. Disponível em: http://www. portcom.intercom.org.br/pdfs/1404297705098614425832679 50533057946044.pdf. Acessoem: 20 abr. 2018.

MARTÍNEZ et.al. Transparencia y acceso a la información en sedes electrónicas municipales. Informação \& Sociedade, João Pessoa, v.28, n.2, p.179-196, maio/ago. 2018. Disponível em: http://www.periodicos.ufpb.br/ojs2/index.php/ies/article/view/31126. Acesso em: 08 mar.2019.

MARTÍNEZ-ÁVILA, D.; PRIETO GORDO, O. ¿Libertad o pragmatismo?: el papel del software libre en la alfabetización tecnológica". In: Conferencia Internacional sobre Brecha Digital e Inclusión Social, 2. 28 al 30 de Octubre de 2009. Madrid: Universidad Carlos III de Madrid, Instituto Universitario Agustín Millares de Documentación y Gestión de la Información, 2009.

SANTOS, J.C.G.; FERNÁNDEZ-MOLINA, J.C.; GUIMARÃES, J.A.C. Direito de acesso àInformação: Uma análise a partir das realidades espanhola e 
brasileira. Informação \& Sociedade, João Pessoa, v.27, n.2, p.49-62, maio/ago. 2017. Disponível em: https://periodicos.ufpb.br/ojs/index.php/ies/article/view/31196. Acesso em: 08 mar. 2019.

SIMÕES, E. Bolsonaro revoga decreto que delegava definição de sigilo a comissionados, 27 fev. 2019. Disponível em: https://extra.globo.com/noticias/brasil/bolsonaro-revoga-decretoque-delegava-definicao-de-sigilo-comissionados23484466.html. Acessoem: 06 mar. 2019.

SHAPIRO, J. J.; HUGHES, S. K. Information Technology as a Liberal Art: Enlightenment Proposals for a New Curriculum. EducomReview, v. 31 n. 2, 1996.

SILVA, J.A. da.Curso de Direito Constitucional Positivo. 17. ed.São Paulo: Malheiros Editores, 2005.

SOUSA, M.R.F.de. O acesso a informações e a contribuição da arquitetura da informação, usabilidade e acessibilidade. Informação \& Sociedade, João Pessoa, v.22, p.65-76, núm.esp., 2012.

STALLMAN, Richard M. Entrevista: Richard M. Stallman / Perspectivas De AñoNuevo,El país, com, 1 de jan. de 2009. Disponível em:https://elpais.com/diario/2009/01/01/ciberpais/1230778287 850215. html. Acesso em: 3 mar. 2019. 